\title{
SERUM CREATININE ASSAY: ENZYMATIC VS KINETIC JAFFE'S METHOD
}

Vijaya Marakala, Avinash S. S, Shivashankara A. R, Malathi M, Arun Kumar

1. Assistant Professor, Department of Biochemistry, Srinivas Institute of Medical Science \& Research Centre, Mukka, Surathkal, Mangalore.

2. Assistant Professor, Department of Biochemistry, Father Muller Medical College, Mangalore.

3. Associate Professor, Department of Biochemistry, Father Muller Medical College, Mangalore.

4. Professor, Department of Biochemistry, Father Muller Medical College, Mangalore.

5. Assistant Professor, Department of Biochemistry, Father Muller Medical College, Mangalore.

\section{CORRESPONDING AUTHOR}

Dr. Vijaya Marakala, Assistant Professor,

Department of Biochemistry,

Srinivas Institute of Medical Science \& Research Centre,

Mukka, Surathkal, Mangalore.

E-mail: vkunder637@gmail.com,

Ph: 00919008165850.

ABSTRACT: BACKGROUND: An enzymatic kit method for the determination of serum creatinine was optimized for use with OLYMPUS AU 400 Auto analyzer and its performance characteristics and practicability compared with kinetic Jaffe-based method. Effects of some common interfering substances like glucose and bilirubin on the kinetic Jaffe's and the enzymatic methods were compared. Method comparison between the enzymatic creatinine method (y) and Jaffe's kinetic method ( $\mathrm{x}$ ) gave the following equation for the normal group: $y=0.97 * x+0.0$ and a coefficient correlation $R$ of 0.98 .There was very good agreement between both the methods as intra class correlation coefficient (ICC) was between 0.81-1.In the presence of high levels of glucose or bilirubin, the coefficient correlation values were 0.99 and 0.89respectively.

KEYWORDS: Creatinine; Enzymatic assay; Kinetic Jaffe's.

INTRODUCTION: Routine clinical biochemistry laboratories use several methods for the estimation of serum and urinary concentrations of creatinine, most of which are based on the Jaffe's reaction described first by Jaffe in 1886. Over the years, the Jaffe's assay has progressed through many phases. There are major analytical problems associated with the use of the Jaffe's reaction, in particular those relating to positive and negative interference by chromogens. More than 50 chromogenic interfering substances have been documented 1 .

Commonly encountered interfering substances of the Jaffe's based methods include glucose, acetoacetate, bilirubin, and cefoxitin ${ }^{2}$. Glucose and bilirubin both inhibit the reaction between creatinine and alkaline picrate. Glucose slowly reduces picric acid to picramate 3 , while bilirubin, under alkaline conditions, is oxidized to biliverdin, causing a decrease in absorbance at $520 \mathrm{~nm}^{4}$. Acetoacetate and Cefoxitin, conversely, react directly with alkaline picrate and cause positive interference. Acetoacetate, in fact, reacts more rapidly with picrate than creatinine ${ }^{5}$.

Enzymatic creatinine assay is widely accepted as one of the most accurate routine methods available at present. Several studies concluded that enzymatic method is suitable as a routine diagnostic laboratory method for the measurement of serum creatinine, particularly for diabetic ketotic patients, neonates, and patients receiving cephalosporins ${ }^{6}$. The enzymatic method exhibits several advantages over Jaffe's based methods-namely, improved specificity, 
smaller sample volume and hence a rapid sample throughput. Glucose, acetoacetate, and Cefoxitin do not interfere with the enzymatic method, although bilirubin causes a negative interference which depends on both creatinine and bilirubin concentrations.

The enzymatic creatinine assay deals effectively with most interfering substances but has a greater cost and shorter shelf-life compared with the kinetic Jaffe's method ${ }^{7}$.In this study an enzymatic kit method for the determination of serum creatinine was optimized for use with OLYMPUS AU 400Auto analyzer and its performance characteristics and practicability were compared with kinetic Jaffe-based method.

The aim of this study was to compare analytical performance and practicability of the enzymatic method and kinetic method for serum creatinine for routine use and to compare the effects of some common interfering substances like glucose and bilirubin on the enzymatic method and kinetic Jaffe's method.

MATERIALS AND METHODS: The present study was conducted in central diagnostic laboratory, department of Biochemistry, Father Muller Medical College Mangalore. We assessed 318 consecutive serum samples obtained for routine clinical care. Creatinine was analyzed both by kinetic Jaffe's and enzymatic method. The Jaffe's method for serum creatinine determination is based on the principle that picric acid in an alkaline medium reacts with creatinine to form an orange coloured complex with the alkaline picrate. Intensity of the colour formed during the fixed time is directly proportional to the amount of creatinine present in the sample. The enzymatic assay for creatinine involves a series of coupled enzymatic reactions including creatininase enzymatic conversion of creatinine into the product creatine which is converted to sarcosine by creatine amidinohydrolase (creatinase), followed by oxidation of sarcosine by sarcosine oxidase producing hydrogen peroxide. In the presence of peroxidase the hydrogen peroxide is quantified at $550 \mathrm{~nm}$ by the formation of a colored dye. All measurements were performed using an Olympus AU 400 analyzer. The 2 levels of quality control materials used in this study were supplied from Biorad. We also estimated Serum Total Bilirubin by azobilirubin method and Fasting Plasma Glucose by hexokinase method of the respective subjects and the data were divided into 3 groups. Group I comprising of 167 samples without interfering substances (Samples having Fasting Plasma Glucose $<126 \mathrm{mg} / \mathrm{dl}$ and Serum Total Bilirubin $\leq 1$ $\mathrm{mg} / \mathrm{dl}$ ).Group II comprising of 33 samples with bilirubin (Samples having Serum Bilirubin $>1 \mathrm{mg} / \mathrm{dl}$ and Fasting Plasma Glucose $<126 \mathrm{mg} / \mathrm{dl}$ ); Group III comprising of 118 samples with glucose (Fasting Plasma Glucose $\geq 126 \mathrm{mg} / \mathrm{dl}$ and Serum Bilirubin $\leq 1 \mathrm{mg} / \mathrm{dl}$ ).

We determined the mean difference between the two methods and analysed the agreement between them. The relationship between the two methods was also compared by regression analysis. The 2 levels of quality control materials were analysed by enzymatic and kinetic Jaffe's methods for precision.

The Statistical Package for the Social Sciences (version 11.0; SPSS) was used for statistical analyses. Data were analyzed by using independent test to assess the significance of difference between the two methods and regression analysis for comparison of methods. Linear regression model was used to establish correlation coefficients.

RESULTS: Mean differences between enzymatic to kinetic Jaffe's methods were $-0.042 \mathrm{mg} / \mathrm{dl}$ in group I; $-0.158 \mathrm{mg} / \mathrm{dl}$ in group II and $-0.116 \mathrm{mg} / \mathrm{dl}$ in group III. Overall mean difference between the two methods was $-0.081 \mathrm{mg} / \mathrm{dl}$. All of the above differences were statistically insignificant ( $p>0.05)$. Intra class correlation coefficients for the agreement between the two methods for 
Group I, Group II, Group III and for all the group together were 0.995, 0.915, 0.997 and 0.995 respectively (Table 1 )

Method comparison between the enzymatic creatinine method (y) and Jaffe's kinetic method (x) by linear regression analysis gave the following equation for all groups together $(n=318)$ gave the following equation: $y=0.97 x-0.04$ with a coefficient correlation $R$ of 0.99 and in the group I $(\mathrm{n}=167): \mathrm{y}=0.97 * \mathrm{x}+0.00$ with a coefficient correlation $\mathrm{R}$ of 0.98 . Regression analysis in group II $(\mathrm{n}=33)$ gave the following equation: $\mathrm{y}=0.80 * \mathrm{x}+0.11$ with a coefficient correlation R of 0.89 and in group III $(n=118)$ gave the following equation: $y=0.98^{*} \mathrm{x}+(-0.08)$ with a coefficient correlation R of 0.98 (Figure 1).

The Quality control analysis of level 1 for precision by Enzymatic method ( $\mathrm{n}=18$ ) yielded a mean, SD,CV and imprecision of $1.45,0.086,5.93$ and 11.86 respectively. The Quality control analysis of level 1 for precision by Kinetic Jaffe's method $(n=79)$ yielded a mean, SD, CV and imprecision of 1.71, 0.082, 4.80 and 9.6 respectively. The Quality control analysis of level 2 for precision by Enzymatic method $(n=18)$ yielded a mean, SD,CV and imprecision of 5.19, 0.365, 7.03 and 14.06 respectively. The Quality control analysis of level 2 for precision by Kinetic Jaffe's method $(\mathrm{n}=79)$ yielded a mean, SD, CV and imprecision of 5.36, 0.246, 4.59 and 9.18 respectively (Table 2).

DISCUSSION: The enzymatic method exhibits advantages over Jaffe's based methods namely, smaller sample volume $(10 \mu \mathrm{L})$ and free of interference from substances such as glucose, acetoacetate and bilirubin. The enzymatic technique yields results directly proportional to the kinetic Jaffe's reaction. Access to enzymatic assays can also be useful when interference from substances such as bilirubin and hemolysis is suspected ${ }^{8}$. On the other hand, a very few compounds may interfere with enzymatic procedures. Interference for enzymatic assays has been reported in case of intravenous fluid contamination of plasma samples from dopamine or dobutamine solutions ${ }^{9}$.The only drug reported to interfere with currently available enzymatic assays at borderline therapeutic concentrations is calcium dobesilate, used to reduce capillary permeability in diabetic retinopathy ${ }^{10}$.

The enzymatic creatinine methods appear to be the only assays giving reliable results when specimens take time to reach the laboratory and blood centrifugation is delayed for $24 \mathrm{~h}$ or more. In a recently published study, delays in sample centrifugation caused false increases in measured creatinine by alkaline picrate assays due to the possible interference effect of some metabolites built up in vitro, such as pyruvate or ketones ${ }^{11}$. A minor disadvantage of the enzymatic method is its relatively high cost.

In our study, estimation of creatinine by enzymatic method showed no statistically significant mean difference $(-0.042)$ with the kinetic Jaffe's method, which is used by several laboratories (including our own center) in samples without glucose and bilirubin interference. In the presence of glucose interference (glucose $>126 \mathrm{mg} / \mathrm{dl}$ ), the samples showed no statistical significant mean difference $(-0.116)$ between enzymatic and kinetic Jaffe's method. This was higher compared to mean difference between the two methods in samples without glucose and bilirubin interference. When bilirubin was present in the serum samples (bilirubin $>1 \mathrm{mg} / \mathrm{dl}$ ), the mean difference between enzymatic and kinetic Jaffe's method was statistically not significant (-0.158). The mean difference between the methods was higher amongst the samples containing bilirubin compared to samples with glucose interference and the samples without glucose and bilirubin interference (Table 1).

Journal of Evolution of Medical and Dental Sciences/Volume1/ Issue4/October - 2012 Page 330 
Method comparison between the enzymatic creatinine method (x) and kinetic Jaffe's method (y) gave the following equation for the whole group of 318 individuals: $y=0.97{ }^{*} \mathrm{x}-0.04$ and a correlation coefficient of 0.99 . The creatinine kinetic Jaffe method gave substantially higher values compared with the enzymatic method. These results are in accordance with several studies that compared an enzymatic method with the kinetic Jaffe method. These results indicate that Jaffe methods, based on an alkaline picrate reaction, overestimate true serum creatinine concentrations due primarily to non-specific protein interference ${ }^{12-14}$.

In this study there was no statistically significance mean difference between both methods in all the groups and the difference was also not clinically significant. The Intra class correlation coefficient between the two methods in group I, Group II, Group III and all the groups together, indicates a very good agreement between Kinetic Jaffe's method and enzymatic method (Table 1). Hence in routine clinical care both the methods can be used (Table 1).

Both the methods showed significant correlation with or without the presence of interfering substances (Figure 1). All the above results indicate a very good comparability between the two methods in all the specified settings with or without the presence of interfering substances like glucose and bilirubin and also when all the groups together were analysed. Analysis of quality controls specimens suggested a comparable precision of enzymatic and Jaffe's methods of creatinine analysis (Table 2). However, the imprecision values obtained in our laboratory settings are much higher than the desirable imprecision of $3 \%$ as per the guidelines ${ }^{15}$.The laboratory is having the mechanism of quality assurance which aims at bringing down the imprecision to the desirable level.

In case of serum creatinine, standardization is of particular importance because of its role in the assessment of renal function and for estimation of glomerular filtration rate ${ }^{16,17}$. Introducing the enzymatic creatinine method to routine laboratory work, instead of the alkaline picrate method, is in accordance with recent recommendations of the Laboratory Working Group of the National Kidney Disease Education Program. This group suggests that the estimated glomerular filtration rate has to be reported using accurate and specific serum creatinine measurements, based on the concept of traceability ${ }^{18}$.

Since the sample volume required is lesser, the throughput is higher, the interfering substances are fewer for the enzymatic method and since there is good agreement and good comparability with the kinetic Jaffe's method, the enzymatic method for estimation can be preferred especially in the setting of neonates, diabetic, ketoacidosis, jaundice and hemolytic samples.

In accordance to ours, another study ${ }^{19}$ evaluated 29 samples with Bilirubin concentrations between 0.1 and $22.7 \mathrm{mg} / \mathrm{dL}(1.7-388.2 \mu \mathrm{mol} / \mathrm{L})$ and did not find a significant difference between 2 methods of creatinine measurement (enzymatic [Ortho Vitros 950] and Jaffe's colorimetric on 2 different analyzers [Roche Hitachi 917 and Dade Di-mension RXL]). In conclusion, enzymatic and kinetic Jaffe's methods of creatinine analysis were comparable with respect to performance in the presence and absence of interfering substances glucose and bilirubin, and imprecision. In this study we employed a small sample size and could test effects of only two interfering substances. Future studies will aim at analyzing the effects of many more interfering substances and validation of two methods by recovery studies, analysis of accuracy, sensitivity and specificity, and evaluation of the methods under allowable imprecision level. Both external and internal quality control programmes will be utilized to increase the accuracy and precision of the creatinine assay methods.

Journal of Evolution of Medical and Dental Sciences/Volume1/ Issue4/October - 2012 Page 331 
Table1. Comparison (by independent ' $t$ ' test) and the agreement between two methods (ICC) of the Serum Creatinine values obtained by Enzymatic Methods and Kinetic Jaffe's method

\begin{tabular}{|c|c|c|c|c|c|}
\hline & & $\begin{array}{l}\text { Mean } \pm \text { SD } \\
(\mathrm{mg} / \mathrm{dl})\end{array}$ & $\begin{array}{l}\text { Mean } \\
\text { differences } \pm \text { SD } \\
(\mathrm{mg} / \mathrm{dl})\end{array}$ & 'p' value & ICC \\
\hline \multirow[t]{2}{*}{$\begin{array}{l}\text { Group I } \\
\text { (Normal) }\end{array}$} & $\begin{array}{l}\text { Enzymatic } \\
(\mathrm{n}=167)\end{array}$ & $1.18 \pm 0.965$ & \multirow{2}{*}{$-0.042 \pm 0.129$} & \multirow{2}{*}{$0.565^{*}$} & \multirow{2}{*}{0.995} \\
\hline & $\begin{array}{l}\text { Kinetic Jaffe's } \\
(n=167)\end{array}$ & $1.23 \pm 0.989$ & & & \\
\hline \multirow[t]{2}{*}{$\begin{array}{l}\text { Group II } \\
\text { (Bilirubin) }\end{array}$} & $\begin{array}{l}\text { Enzymatic } \\
(\mathrm{n}=33)\end{array}$ & $1.20 \pm 0.452$ & \multirow{2}{*}{$-0.158 \pm 0.228$} & \multirow[b]{2}{*}{$0.186^{*}$} & \multirow{2}{*}{0.915} \\
\hline & $\begin{array}{l}\text { Kinetic Jaffe's } \\
(\mathrm{n}=33)\end{array}$ & $1.35 \pm 0.503$ & & & \\
\hline \multirow[t]{2}{*}{$\begin{array}{l}\text { Group III } \\
\text { (Glucose) }\end{array}$} & $\begin{array}{l}\text { Enzymatic } \\
(\mathrm{n}=118)\end{array}$ & $1.52 \pm 1.581$ & \multirow{2}{*}{$-0.116 \pm 0.134$} & \multirow[t]{2}{*}{$0.577^{*}$} & \multirow{2}{*}{0.997} \\
\hline & $\begin{array}{l}\text { Kinetic Jaffe's } \\
(n=118)\end{array}$ & $1.63 \pm 1.610$ & & & \\
\hline \multirow[t]{2}{*}{$\begin{array}{l}\text { All Groups } \\
\text { (Total) }\end{array}$} & $\begin{array}{l}\text { Enzymatic } \\
(n=318)\end{array}$ & $1.31 \pm 1.207$ & \multirow[b]{2}{*}{$-0.081 \pm 0.150$} & \multirow[t]{2}{*}{$0.401^{*}$} & \multirow[b]{2}{*}{0.995} \\
\hline & $\begin{array}{l}\text { Kinetic Jaffe's } \\
(\mathrm{n}=318)\end{array}$ & $1.39 \pm 1.239$ & & & \\
\hline
\end{tabular}

* $\mathrm{p}>0.05$ (' $\mathrm{p}$ ' values for mean differences between two methods by independent ' $\mathrm{t}$ ' test ICC - Intra class correlation coefficient- used to assess the agreement between two methods. ICC: $<0.2$ Poor agreement, 0.21 - 0.4 Fair agreement, 0.41 - 0.6 Moderate agreement, $0.61-0.8$ Good agreement and $0.81-1$ Very good agreement.

Table 2. Precision analysis of Kinetic Jaffe's and Enzymatic Methods with Quality Controls Levels 1 and 2

\begin{tabular}{|l|l|l|l|l|}
\hline \multicolumn{2}{|l|}{ Level 1 Quality Control } & \multicolumn{2}{l|}{ Level 2 Quality Control } \\
\hline & $\begin{array}{l}\text { Creatinine } \\
(\mathrm{mg} / \mathrm{dl})\end{array}$ & $\begin{array}{l}\text { Creatinine } \\
(\mathrm{mg} / \mathrm{dl}) \text { Enzymatic } \\
\text { Kinetic Jaffe's } \\
(\mathrm{n}=79)\end{array}$ & $\begin{array}{l}\text { Creatinine } \\
(\mathrm{mg} / \mathrm{dl})\end{array}$ & $\begin{array}{l}\text { Creatinine } \\
\text { (mg/dl)Enzymatic } \\
\text { Kinetic Jaffe's } \\
(\mathrm{n}=79)\end{array}$ \\
\hline Mean & 1.71 & 1.45 & 5.36 & 5.19 \\
\hline SD & 0.082 & 0.086 & 0.246 & 0.365 \\
\hline $\mathrm{CV}(\%)$ & 4.80 & 5.93 & 4.59 & 7.03 \\
\hline
\end{tabular}

Journal of Evolution of Medical and Dental Sciences/Volume1/ Issue4/October - 2012 Page 332 
Figure 1: Method comparison by linear regression between Enzymatic and Kinetic Jaffe's method.
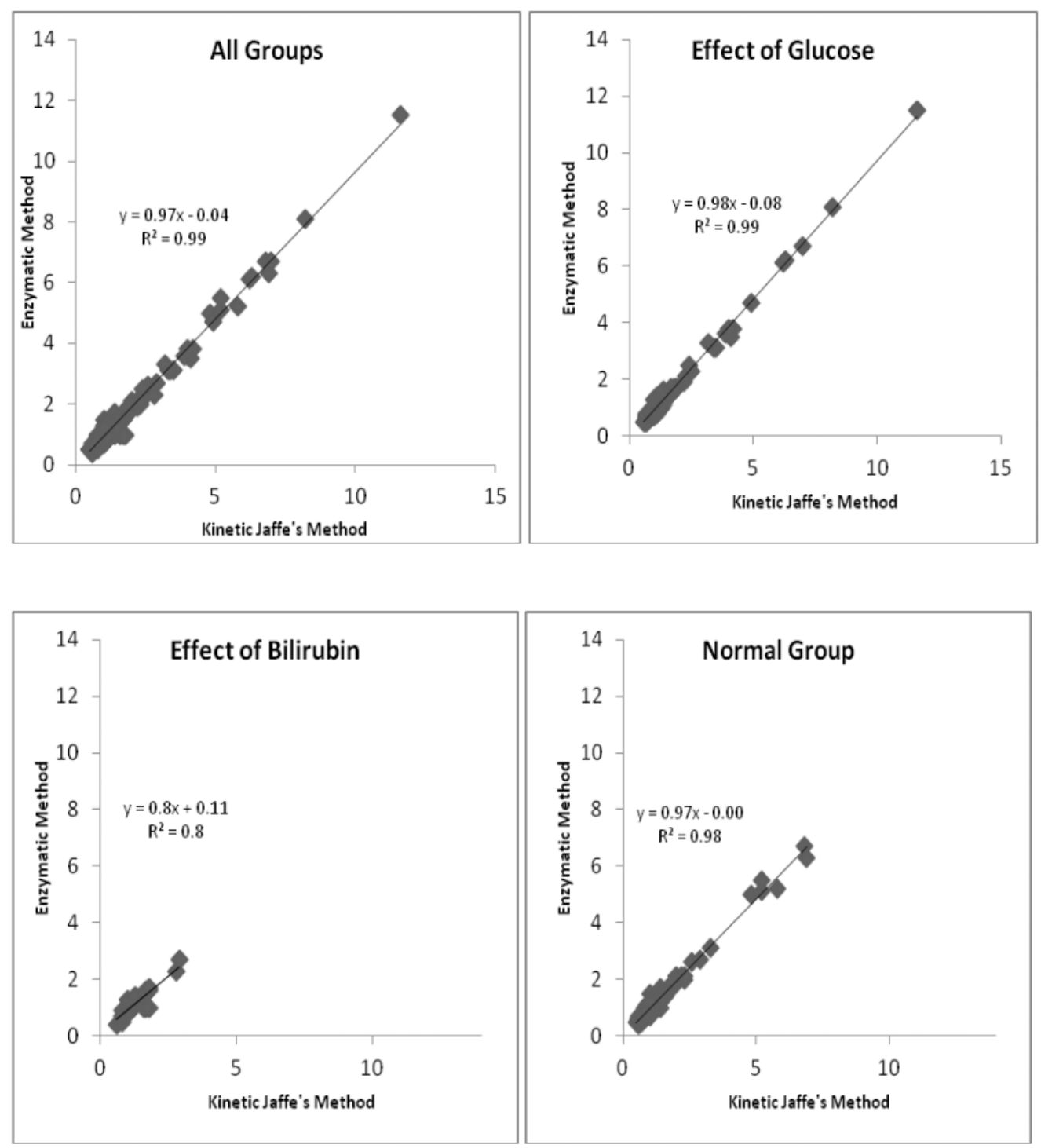

\section{REFERENCES:}

1. Cook JGH. Factors influencing the assay of creatinine. Ann Clin Biochem 1975;12:219-32.

2. Spencer K. Analytical reviews in clinical biochemistry: the estimation of creatinine. Ann Clin Biochem 1986;23:1-25.

3. Bowers LD, Wong ET. Kinetic creatinine assays. II. A critical evaluation and review. Clin Chem 1980;26:555-61.

4. Knapp ML, Hadid 0. Investigations into negative interference by jaundiced plasma in kinetic Jaffe methods for plasma creatinine determinations. Ann Clin Biochem 1987;24:85-97. 
5. Gerard SK, Khayam-Bashi H. Characterization of creatinine error in ketotic patients. Am J Clin Pathol 1985; 84:659-64.

6. R M Jacobs, J H Lumsden, J A Taylor, and E Grift Effects of interferents on the kinetic Jaffé reaction and an enzymatic colorimetric test for serum creatinine concentration determination in cats, cows, dogs and horses. Can J Vet Res. 1991 April; 55(2): 150-54.

7. H Crocker, M D S Shephard, G H White Evaluation of an enzymatic method for determining creatinine in plasma.J Clin Pathol 1988;41:576-81

8. Peake M, Whiting M. Measurement of serum creatinine -Current status and future goals. Clin Biochem Rev 2006;27:173-84

9. Karon BD, Daly TM, Scott MG. Mechanisms of dopamine and dobutamine interference in biochemical tests that use peroxide and peroxidase to generate chromophore. Clin Chem 1998;44:155-60.

10. Perrone RD, Madias NE, Levey AS. Serum creatinine as an index of renal function: new insights into old concepts.Clin Chem 1992;38:1933-53

11. Shepherd J, Warner MH, Kilpatrick ES. Stability of creatinine with delayed separation of whole blood and implications for eGFR. Ann Clin Biochem2007;44:384-87.

12. Panteghini M on behalf of the IFCC Scientific Division. Enzy-matic assays for creatinine: time for action. Clin Chem LabMed. 2008; 46:567-72.

13. Delanghe JR, Cobbaert C, Galteau MM, Harmoinen A, JansenR, Kruse R, et al. Trueness verification of actual creatinineassays in the European market demonstrates a disappointingvariability that needs substantial improvement. Clin Chem LabMed. 2008; 46:1319-25.

14. Chromy V, Rozkosna K, Sedla K P. Determination of serum creatinine by Jaffe method and how to calibrate to eliminatematrix interference problems. Clin Chem Lab Med. 2008; 46:1127-33.

15. Ricos C, Alvarez V, Cava F, Garcia-Lario JV, Hernandez A, Jimenez CV, Mininchela J, Perich C, Simon M. "Current databases on biologic variation: pros, cons and progress" Scand J Clin Lab Invest 1999;59:491-500

16. Fuentes-Arderiu X, A'lvarez-Funes V, Coca-Fa 'bregas L, Cruz-Placer M, Dı'az-Ferna 'ndez J, Herrero-Bernal $\mathrm{P}$, et al. Multi-centre physiological reference values for the concentration of creatininium in plasma and diagnostic specificity of glomerular filtration rate estimated with the MDRD equation. Clin ChemLab Med 2007;45:531-4.

17. Van Biesen W, Vanholder R, Veys N, Verbeke F, Delanghe J, De Bacquer D, et al. The importance of standardization of creatinine in the implementation of guidelines and recommen-dations for CKD: implications for CKD management pro-grammes. Nephrol Dial Transplant 2006;21:77-83

18. Myers GL, Miller WG, Coresh J, Fleming J, Greenberg N, Greene T, et al. Recommendations for improving serum creat-inine measurement: a report from the Laboratory Working Group of the National Kidney Disease Education Program. Clin Chem 2006; 52:5-18.

19. Trotter JF, Brimhall B, Arjal R, Phillips C. Specific labo-ratory methodologies achieve higher model for endstageliver disease (MELD) scores for patients listed for liver transplantation. Liver Transpl 2004;10:995-1000 